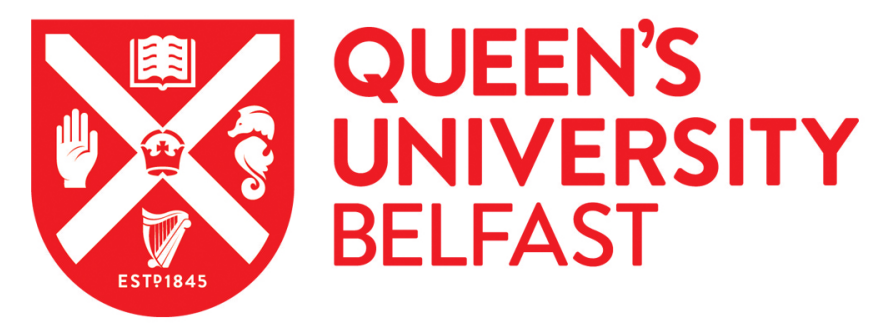

\title{
A novel approach to the computation of the maximal controlled invariant set for constrained linear systems
}

Athanasopoulos, N., \& Bitsoris, G. (2009). A novel approach to the computation of the maximal controlled invariant set for constrained linear systems. In Proceedings of the 2009 European Control Conference Institute of Electrical and Electronics Engineers Inc.. http://ieeexplore.ieee.org/abstract/document/7074885/

Published in:

Proceedings of the 2009 European Control Conference

Document Version:

Peer reviewed version

Queen's University Belfast - Research Portal:

Link to publication record in Queen's University Belfast Research Portal

\section{General rights}

Copyright for the publications made accessible via the Queen's University Belfast Research Portal is retained by the author(s) and / or other copyright owners and it is a condition of accessing these publications that users recognise and abide by the legal requirements associated with these rights.

Take down policy

The Research Portal is Queen's institutional repository that provides access to Queen's research output. Every effort has been made to ensure that content in the Research Portal does not infringe any person's rights, or applicable UK laws. If you discover content in the Research Portal that you believe breaches copyright or violates any law, please contact openaccess@qub.ac.uk. 


\title{
A novel approach to the computation of the maximal controlled invariant set for constrained linear systems
}

\author{
Nikolaos Athanasopoulos and George Bitsoris
}

\begin{abstract}
In this paper, the problem of the determination of the maximal controlled invariant set of linear systems subject to polyhedral input and state constraints, together with the corresponding state-feedback control law is investigated. Instead of computing one-step reachable sets or maximizing the volume of a specific invariant set, the proposed method consists of the iterative expansion of an initial "small" invariant set by adding new vertices to its convex hull. This is achieved by minimizing the distance of each new vertex from the vertices of the polyhedral set defining the state constraints. This approach, established for both continuous-time and discrete-time systems, does not require invertibility of matrix $A$, open-loop stability or symmetry of the constraints.
\end{abstract}

\section{INTRODUCTION}

Two major approaches have been developed to tackle the problem of regulation of systems with state and control constraints: model predictive control and set theoretic methods. In model predictive control [1], [3], constraints are naturally embedded in the optimization procedure. In set theoretic methods [2], the constraints are related to sets characterized by properties that ensure constraint satisfaction. For both approaches, the estimation of the maximal region of the state space where the system can operate without violating the constraints is a very important problem. This problem is related to the determination of controlled invariant sets. These sets (with the exception of [4]) may be ellipsoids or polytopes.

The usual method to determine the exact or an estimate of the maximal invariant set is through one-step reachable sets [5],[6],[7],[8],[9],[10],[11]. Specifically, Gutman and Chwikel [5] proposed an algorithm based on vertex computation which produces the maximal $\Omega$ invariant set. In [6] the notion of output and maximal output admissible sets was studied in a more general framework and algorithms based on the computation of $\mathrm{N}$-reachable sets were proposed. Lassere [9] proposed an approach to determine the Nreachable and controllable set by only checking the unstable subspace of the autonomous system . The problem of finding a stabilizing solution with an assigned initial condition set was studied in [16],[11]. In [11] the authors proposed a forward algorithm which finds a stabilizing solution for trajectories starting from the vertices of the assigned set and a backward algorithm based on the N-controllable set. Dorea and Hennet [7],[8] proposed an algorithm based on

This work was supported in part by the Greek State Scholarships Foundation

N. Athanasopoulos and G. Bitsoris are with the Electrical and Computer Engineering Department, University of Patras, Rio 26500, Achaia, Greece. e-mail nathanaseece.upatras.gr, bitsoris@ece. upatras.gr the half plane representation of sets by formulating algebraic conditions of (A,B) invariance. In [12] an LMI approach was used for the enlargement of the domain of attraction using lifting techniques. The terminal invariant set in [13] was enlarged using a linear programming approach. In [14] convex optimization problems are formulated for the enlargement of the stability region. In [15] a tuning parameter for the enlargement of the positively invariant set was introduced.

In this paper, a new approach to the estimation of the maximal controlled invariant set and to the determination of a corresponding state-feedback control law is developed. Instead of trying to compute one-step reachable sets, the enlargment of an initially "small" polyhedral controlled invariant set is carried out iteratively by adding at each step a new vertex to its convex hull. This is achieved by minimizing at each step the distance of the new vertex from the vertices of the assigned initial condition set. This method does not require invertibility of matrix A, open-loop stability, controllability of $(\mathrm{A}, \mathrm{B})$ or symmetry of the constraint sets . It can be applied to both discrete-time and continuous-time linear systems and can also be extended to the determination of controlled invariant sets with linear state-feedback control laws.

\section{Problem Statement}

Throughout the paper, $\mathbb{R}^{n}$ denotes the real $n$-space and $\mathbb{R}^{m \times n}$ denotes the set of real $m \times n$ matrices. The elements of a real matrix $P \in \mathbb{R}^{m \times n}$ are denoted by $p_{i j} . P \geq 0$ is a matrix with nonnegative elements. For vectors $a, b$ relation $a \leq b$ holds componentwise. For two sets $S$ and $Q$, $S \backslash Q$ denotes their set difference i.e. the set that contains all elements of $S$ that do not belong to $Q$. For a set $S, \operatorname{int}(S)$ denotes the interior of $S$. Given $q$ points $v^{1}, \ldots, v^{q}$ defined on the real $n$-space $\mathbb{R}^{n}, S=\operatorname{conv}\left\{v^{1}, \ldots, v^{q}\right\}$ denotes the convex hull of $v^{1}, \ldots, v^{q}$.

We consider both continuous-time and discrete-time linear systems. Continuous-time systems are described by differential equations of the form

$$
\dot{x}(t)=A x(t)+B u(t)
$$

while discrete-time systems are described by difference equations of the form

$$
x(t+1)=A x(t)+B u(t)
$$

where $x \in \mathbb{R}^{n}$ is the state vector, $u \in \mathbb{R}^{m}$ is the input vector, $A \in \mathbb{R}^{n \times n}, B \in \mathbb{R}^{n \times m}$ and $t$ is the time variable belonging to the set $[0, \infty)$ in the case of continuous-time systems or 
to the set of nonnegative integers in the case of discrete-time systems.

Although the method can deal with any polyhedral input constraint set, for simplicity of the presentation the control variable $u$ is constrained to belong to a set $U \subseteq \mathbb{R}^{m}$ defined by the relation

$$
U=\left\{u \in \mathbb{R}^{m}:-\underline{u} \leq u \leq \bar{u}\right\}
$$

where $\underline{u}$ and $\bar{u}$ are vectors with nonnegative components. Thus, $-\underline{u}$ and $\bar{u}$ represent the lower and upper bounds of the control variables.

Definition 1: A subset $S \subset \mathbb{R}^{n}$ of the state space is said to be controlled invariant w.r.t. system (2) or (3) if and only if there exists a feedback control law $u=f(x) \in U$, such that $x_{0} \in S$ implies $x\left(t ; x_{0}\right) \in S$ for all $t \geq 0$.

Definition 2: Given a set $S \subset \mathbb{R}^{n}$, a subset $S^{M} \subseteq S$ is said to be the maximal controlled invariant set if and only if it is controlled invariant and contains all controlled invariant sets contained in $S$ for a specific input constraint set $U$.

Definition 3: A subset $S \subset \mathbb{R}^{n}$ of the state space is said to be positively invariant w.r.t. to an autonomous system if and only if for any initial state $x_{0} \in S$ the corresponding trajectory $x\left(t ; x_{0}\right)$ satisies relation $x\left(t ; x_{0}\right) \in S$ for all $t \geq 0$.

In this paper we study the controlled invariance of bounded convex polyhedral subsets $S$ of the state space $\mathbb{R}^{n}$ containing the origin as an interior point. Such polyhedral sets are represented as

$$
S=\left\{x \in \mathbb{R}^{n}: G x \leq w\right\}
$$

with $G \in \mathbb{R}^{r \times n}$ and $w \in \mathbb{R}^{r}, w>0$. A bounded convex polyhedral set can also be represented as the convex hull of its vertices $v^{1}, \ldots, v^{q}$, i.e.

$$
S=\operatorname{conv}\left\{v^{1}, \ldots, v^{q}\right\}
$$

The problem to be investigated is formulated as follows: Given a linear system (1) or (2) and state and input constraint sets $S$ and $U$ respectively, determine an estimate $S_{e}$ of the maximal controlled invariant set $S^{M} \subseteq S$ and the corresponding control law $u_{e}(x)$ making set $S_{e}$ both positively invariant and domain of attraction of the origin for the resulting closed-loop system.

\section{PRELIMINARIES}

It is known [16] that a polyhedral set represented by (4) is positively invariant set of an autonomous linear discretetime system $x(t+1)=A x(t)$ if and only if there exists a nonnegative matrix $H \in \mathbb{R}^{r \times r}$ such that

$$
\begin{gathered}
G A=H G \\
H w \leq w .
\end{gathered}
$$

If the polyhedral set $S$ is represented as the convex hull of its vertices (5) then ([17],[18]) its positive invariance w.r.t. to an autonomous linear discrete-time system $x(t+1)=A x(t)$ is equivalent to the existence of a nonnegative matrix $P \in \mathbb{R}^{q \times q}$ such that

$$
\begin{aligned}
& A V=V P \\
& e^{T} P \leq e^{T}
\end{aligned}
$$

where $V \in \mathbb{R}^{n \times q}$ is the matrix with columns $V=\left[\begin{array}{llll}v^{1} & v^{2} & \cdots & v^{q}\end{array}\right]$ and $e \in \mathbb{R}^{q}, e=$ $\left[\begin{array}{llll}1 & 1 & \cdots & 1\end{array}\right]^{T}$.

In the case of autonomous linear continuous-time systems $\dot{x}(t)=A x(t)$ [19], the positive invariance of a polyhedral set represented by (4) is equivalent to the existence of a matrix $H \in \mathbb{R}^{r \times r}$ with nonnegative off-diagonal entries such that

$$
\begin{gathered}
G A=H G \\
H w \leq 0 .
\end{gathered}
$$

If the polyhedral set $S$ is represented as the convex hull of its vertices (5) then its positive invariance w.r.t. to an autonomous linear continuous-time system $\dot{x}(t)=A x(t)$ is equivalent to the existence of matrix $P \in \mathbb{R}^{q x q}$ with nonnegative off-diagonal entries such that

$$
\begin{aligned}
& A V=V P \\
& e^{T} P \leq 0 .
\end{aligned}
$$

Equivalent conditions to (10)-(11) and (12)-(13) have also been established by [20] and [21] respectively.

Given control constraints (3), a polyhedral set $S=$ $\operatorname{conv}\left\{v^{1}, \ldots, v^{m}\right\}$ is controlled invariant w.r.t. the linear discrete-time system (2) if and only if [21] there exist $u^{i}$ $\in U, i=1, \ldots, q$ and a nonnegative matrix $P \in \mathbb{R}^{q \times q}$ such that

$$
\begin{gathered}
A V+B\left[\begin{array}{llll}
u^{1} & u^{2} & \cdots & u^{q}
\end{array}\right]=V P \\
e^{T} P \leq e^{T}
\end{gathered}
$$

Moreover, set $S$ can be a domain of attraction if instead of (15) the following inequalities are satisfied:

$$
\begin{gathered}
e^{T} P \leq \varepsilon e^{T} \\
0<\varepsilon<1 .
\end{gathered}
$$

The polyhedral set $S=\operatorname{conv}\left\{v^{1}, \ldots, v^{m}\right\}$ is controlled invariant w.r.t. continuous-time linear system (1) if and only if there exist $u^{i}, i=1, \ldots, q$ and a matrix $P \in \mathbb{R}^{q \times q}$ with nonnegative off-diagonal elements such that

$$
\begin{gathered}
A V+B\left[\begin{array}{cccc}
u^{1} & u^{2} & \cdots & u^{q}
\end{array}\right]=V P \\
e^{T} P \leq 0 .
\end{gathered}
$$

Equivalent conditions have also been established by Blanchini and Miani [21]. $S$ is also a domain of attraction if inequality (19) is replaced by the following conditions:

$$
\begin{gathered}
e^{T} P \leq \varepsilon e^{T} \\
\varepsilon<0 .
\end{gathered}
$$

If conditions (14)-(15) or (18)-(19) are satisfied for discrete-time and continuous-time systems respectively, then 
there exist state-feedback control laws rendering set $S$ positively invariant. A possible approach to the determination of such state-feedback control laws $u(x)$ consists in first solving the optimization problem

$$
\min _{u^{1}, \ldots, u^{q}, P, \varepsilon}\{\varepsilon\}
$$

under constraints

$$
-\underline{u} \leq u^{i} \leq \bar{u}, \quad i=1,2, \ldots, q
$$

and (14)-(15) or (18)-(19) for a discrete-time or a continuoustime system respectively. Then a solution to this problem can be obtained [22], [23] by setting

$$
u(x)=\sum_{i=1}^{q} \lambda_{i}(x) u^{i}
$$

where $\lambda_{i}(x), \quad i=1,2, \ldots, q$ are nonnegative real numbers such that $\sum_{i=1}^{q} \lambda_{i}(x) \leq 1$ and $x=\sum_{i=1}^{q} \lambda_{i}(x) v^{i}$.

\section{Main Results}

We now consider the case when set $S$ cannot be controlled invariant, that is the case when the above conditions of controlled invariance are not satisfied. We assume that the unconstrained system under consideration satisfies conditions guaranteeing the existence of a linear state-feedback control law so that the resulting closed-loop system possesses polyhedral invariant sets. Then, by contraction, it is always possible to determine a sufficiently "small" polyhedral set $S_{0}=\operatorname{conv}\left\{v_{0}^{1}, . ., v_{0}^{m_{0}}\right\}, S_{0} \subset S$, which is controlled invariant. The goal is to enlarge this set and, if possible, to derive the maximal controlled invariant set $S^{M}$ included in $S$.

Most methods for enlarging a polyhedral controlled invariant set are based upon the one-step reachable sets. These approaches, developed for discrete-time systems, provide polyhedral controlled invariant sets with unacceptably big number of vertices and cannot be extended to continuoustime systems. In addition, by this approach only nonlinear control laws can be obtained.

In this section, a systematic method of recursively increasing the volume of a controlled invariant subset of $S$ is described. Starting from a polyhedral controlled invariant set $S_{j}=\operatorname{conv}\left\{v_{j}^{1}, . ., v_{j}^{q_{j}}\right\}$, at each step a new polyhedral controlled invariant set $S_{j+1}=\operatorname{conv}\left\{v_{j+1}^{1}, . .,, v_{j+1}^{q_{j+1}}\right\}=$ $\operatorname{conv}\left\{v_{j}^{1}, \ldots, v_{j}^{q_{j}}, v^{*}\right\}$ with $v_{j+1}^{i}=v_{j}^{i}, i=1,2, \ldots, q_{j}$ is constructed. It is worth mentioning that adding a vertex at each step in the convex hull of set $S_{j}$ does not necessarily increase the complexity of the representation of the set. The new vertex $v_{j+1}^{q_{j+1}}=v^{*}$ is determined by minimizing its distance from a point $v^{c h}$ belonging to $S \backslash S_{j}$. In the sequel, we develop this approach for the discrete-time case:

Step 0. The algorithm starts with the determination of an arbitrarily "small" polyhedral controlled invariant set $S_{0} \subset$ $S$,

$$
S_{0}=\operatorname{conv}\left\{v_{0}^{1}, . ., v_{0}^{q_{0}}\right\}, v_{0}^{i} \in \mathbb{R}^{n}, i=1, \ldots, q_{0}
$$

and of a set of control vectors $u_{0}^{i} \in U, i=1, \ldots, q_{0}$ corresponding to each vertex $v_{0}^{i} \in \mathbb{R}^{n}, i=1, \ldots, q_{0}$ of $S_{0}$.
Step 1. At this step, we have already computed a polyhedral controlled invariant set $S_{j}$

$$
S_{j}=\operatorname{conv}\left\{v_{j}^{1}, . ., v_{j}^{q_{j}}\right\}, v_{j}^{i} \in \mathbb{R}^{n}, i=1, \ldots, q_{j}
$$

and a corresponding set of control vectors $u_{j}^{i} \in U, i=$ $1, \ldots, q_{j}$.

We choose a point $v^{c h} \in S$ outside set $S_{j}$ and solve the following optimization problem:

$$
\min _{v^{*}, p, u^{*}, \varepsilon}\left\{\left\|v^{*}-v^{c h}\right\|_{\infty}\right\}
$$

subject to

$$
\begin{gathered}
A v^{*}+B u^{*}=\sum_{i=1}^{q_{j}} p_{i} v_{j}^{i}+p_{q_{j}+1} v^{*} \\
p_{i} \geq 0, i=1, \ldots, q_{j}+1 \\
\sum_{i=1}^{q_{j}+1} p_{i} \leq \varepsilon \\
0<\varepsilon \leq 1 \\
G v^{*} \leq w \\
-\underline{u} \leq u^{*} \leq \bar{u}
\end{gathered}
$$

where $G$ and $w$ correspond to the half-plane representation of set $S$. The optimization criterion can always be made linear because it is equivalent to

$$
\min _{v^{*}, p, u^{*}, \varepsilon, \delta}\{\delta\}
$$

with the additional constraints :

$$
-\delta \leq v_{i}^{*}-v_{i}^{c h} \leq \delta, i=1, . ., n .
$$

This optimization problem can be reduced to a sequence of linear programming problems by solving each time the problem with $p_{q_{j}+1}=a \in[0,1)$. Among all points $v^{*}$ produced by the solution of the LP problems, we choose the one closest to $v^{\text {ch }}$.

If the optimal $v^{*}$ does not belong to $S_{j}$, then, setting $v_{j+1}^{q_{j+1}}=v^{*}$ and $u_{j+1}^{q_{j+1}}=u^{*}$, we construct the following set

$$
S_{j+1}=\operatorname{conv}\left\{v_{j}^{1}, \ldots, v_{j}^{q_{j}}, v^{*}\right\}=\operatorname{conv}\left\{v_{j+1}^{1}, \ldots, v_{j+1}^{q_{j+1}}\right\} .
$$

together with the set of control vectors $\left\{u_{j+1}^{1}, \ldots, u_{j+1}^{q_{j+1}}\right\}$. Relations (23)-(26) imply the positive invariance and attractivity of $S_{j+1}$ (when $\varepsilon$ is strictly less than 1) while (27) and (28) guarantee constraint satisfaction. It is clear that $S_{j+1} \supset S_{j}$. The corresponding control law that makes set $S_{j+1}$ both positively invariant and domain of attraction of the closed-loop system is

$$
u(x)=\sum_{i=1}^{q_{j+1}} \lambda_{i}(x) u_{j+1}^{i}
$$

Then setting $S_{j}=S_{j+1}$ we repeat this procedure to determine a new "larger" polyhedral controlled invariant set. 
If the optimal $v^{*}$ belongs to $S_{j}$ we set

$$
E_{i}=\left\{x \in \mathbb{R}^{n}:\left\|x-v^{c h}\right\|_{\infty}<d^{*}\right\} \cap S
$$

$d^{*}$ being the optimal value of criterion (22), and we proceed to Step 2. Set $E_{i}$ consists of points that have already been tested for "expansion" of set $S_{j}$ with no success.

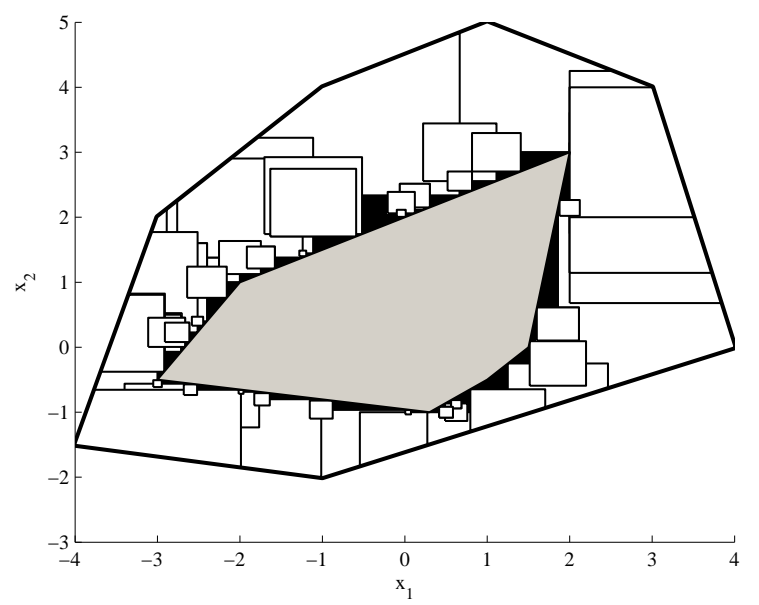

Fig. 1. Sets $S$ and $E_{i}$ (white), $S_{j}$ (gray), and set $T$ remaining to be checked for the expansions of $S_{j}$ (black).

Step 2. Since the optimization procedure has not produced a vector $v^{*} \notin S_{j}$, set $E_{i}$ is excluded from future search at this stage. If $E$, which is the set union of all sets $E_{i}$ excluded in past iterations, satisfies relation $E \cup S_{j} \neq S$, then we choose another point $v^{c h} \in S \backslash\left(S_{j} U E\right)$ and repeat step 1. Otherwise, i.e $E \cup S_{j}=S$, the algorithm terminates and the maximal controlled invariant set is $S_{\max }=S_{j}$.

The choice of vectors of $v^{c h}$ at each iteration of this algorithm is crucial, because all points of set $S \backslash S_{j}$ have to be tested for possible "expansion" of set $S_{j}$. In this paper, we choose the initial vectors $v^{c h}$ to be the $q$ vertices of $S$. After $q$ unsuccessful iterations we construct sets $E_{i}=Q_{i} \cap S$, $i=1, \ldots, q$, where $Q_{i}=\left\{x \in \mathbb{R}^{n}:\left\|x-v_{i}^{c h}\right\|_{\infty} \leq\right.$ $\left.d_{i}^{*}\right\}$. The vertex-representation of every set $Q_{i}$ is $Q_{i}=$ $\operatorname{conv}\left\{r_{1}, \ldots, r_{2^{n}}\right\}$, where $r_{j}=v_{i}^{c h}+d_{i} D_{j}, j=1, \ldots, 2^{n}$ and $D \in \mathbb{R}^{n x 2^{n}}$ a matrix with columns all $2^{n}$ distinct vectors with elements equal to 1 or -1 . Then, we compute set $T=S \backslash\left(E_{1} \cup \ldots \cup E_{q} \cup S_{j}\right)$. The new vectors $v^{c h}$ are the extreme points of set $T$. These new vectors will produce new sets $E_{i}, i=q+1, \ldots, k$. Set $T$ will be updated to $T=S \backslash\left(E_{1} \cup \ldots \cup E_{k_{1}} \cup S_{j}\right)$. In Fig.1, set $T$ as well as sets $E_{i}, i=1, \ldots, k$ are shown. This procedure will continue until $T=\varnothing$.

The algorithm converges to the maximal controlled invariant set because otherwise there would exist another controlled invariant set $W \supset S_{\max }=\operatorname{conv}\left\{v_{\max }^{1}, \ldots, v_{\max }^{q_{\max }}\right\}$. Then, there would exist a point $x_{0} \in W$ such that $x_{0} \notin$ $S_{\max }$. This would imply the existence of a time instant $M>1$ such that $x\left(M ; x_{0}\right) \in S_{\max }$ while $x\left(M-1 ; x_{0}\right) \notin$ $S_{\max }$. This in turn would imply the existence of nonnegative scalars $p_{i}, i=1, . ., q_{\max }+1, \sum_{i=1}^{q_{\max }+1} p_{i} \leq 1$ such that $x\left(1 ; x\left(M-1 ; x_{0}\right)\right)=\sum_{i=1}^{q_{\max }} p_{i} v_{\max }^{i}+p_{q_{\max }+1} x\left(M-1 ; x_{0}\right)$

Consequently, the set

$$
S^{i}=\operatorname{conv}\left(v_{\max }^{1}, \ldots, v_{\max }^{m_{\max }}, x\left(M-1 ; x_{0}\right)\right) .
$$

would be controlled invariant. This set however would have been determined in step 1, thus contradicting the hypothesis that $x\left(M-1 ; x_{0}\right) \notin S_{\max }$.

In addition, it can be clearly seen that the algorithm converges to the maximal controlled invariant set independently of the choice of initial set $S_{0}$.

\section{A. Determination of linear state-feedback control laws}

The algorithm described above can be modified in order to produce a polyhedral controlled invariant set together with a linear state- feedback control law making this set positively invariant. To this end, it is sufficient to replace the optimization problem in step 1 , by the following nonlinear programming problem:

$$
\min _{v^{*}, P, K_{j+1}, \varepsilon_{k}}\left\{\left\|v^{*}-v^{c h}\right\|_{\infty}\right\}
$$

subject to

$$
\begin{gathered}
\left(A+B K_{j+1}\right) V=V P \\
P \geq 0
\end{gathered}
$$

$$
\begin{gathered}
\sum_{i=1}^{q_{j}+1} p_{i k} \leq \varepsilon_{k}, \quad k=1, . ., q_{j}+1 \\
\varepsilon_{k}<1 \quad k=1, . ., q_{j}+1 \\
G_{s} v^{*} \leq w_{s} \\
-\underline{u} \leq K_{j+1} v^{j} \leq \bar{u}, \quad j=1, . ., q_{j} \\
-\underline{u} \leq K_{j+1} v^{*} \leq \bar{u}
\end{gathered}
$$

where $V \in \mathbb{R}^{n \times\left(q_{j}+1\right)}, V=\left[\begin{array}{llll}v^{1} & \cdots & u^{q} & v^{*}\end{array}\right]$ and $P \in \mathbb{R}^{\left(q_{j}+1\right) \times\left(q_{j}+1\right)}$ is a matrix with nonnegative elements. This nonlinear programming problem is always feasible. The algorithm converges although it is not guaranteed that the maximal controlled invariant set is reached.

\section{B. Continuous time systems.}

We can apply the same algorithm with slightly different constraints:

$$
\min _{v^{*}, p, u^{*}, \varepsilon}\left\{\left\|v^{*}-v^{c h}\right\|_{\infty}\right\}
$$

subject to

$$
\begin{gathered}
A v^{*}+B u^{*}=\sum_{i=1}^{q_{j}} p_{i} v_{j}^{i}+p_{q_{j}+1} v^{*} \\
p_{i} \geq 0, i=1, \ldots, q_{j}+1 \\
\sum_{i=1}^{q_{j}+1} p_{i} \leq-\varepsilon
\end{gathered}
$$




$$
\begin{gathered}
\varepsilon>0 \\
G_{s} v^{*} \leq w_{s} \\
-\underline{u} \leq u^{*} \leq \bar{u}
\end{gathered}
$$

For the derivation of a linear state-feedback control law the optimization problem in step 1 becomes:

$$
\min _{v^{*}, P, K_{j+1}, \varepsilon_{k}}\left\{\left\|v^{*}-v^{c h}\right\|_{\infty}\right\}
$$

subject to

$$
\begin{gathered}
\left(A+B K_{j+1}\right) V=V P \\
P \geq 0 \\
\sum_{i=1}^{q_{j}+1} p_{i k} \leq-\varepsilon_{k} \\
\varepsilon_{k}>0 \\
k=1, . ., q_{j}+1 \\
G_{s} v^{*} \leq w_{s} \\
-\underline{u} \leq K_{j+1} v^{j} \leq \bar{u}, j=1, . ., q_{j} \\
-\underline{u} \leq K_{j+1} v^{*} \leq \bar{u}
\end{gathered}
$$

where $V \in \mathbb{R}^{n \times\left(q_{j}+1\right)}, V=\left[\begin{array}{llll}v^{1} & \cdots & u^{q} & v^{*}\end{array}\right]$.

\section{NumERICAL EXAMPLE}

We consider the following linear discrete time system [24]

$$
A=\left[\begin{array}{cc}
1 & 0.1 \\
0 & 0.98
\end{array}\right], \quad B=\left[\begin{array}{c}
0 \\
0.98
\end{array}\right]
$$

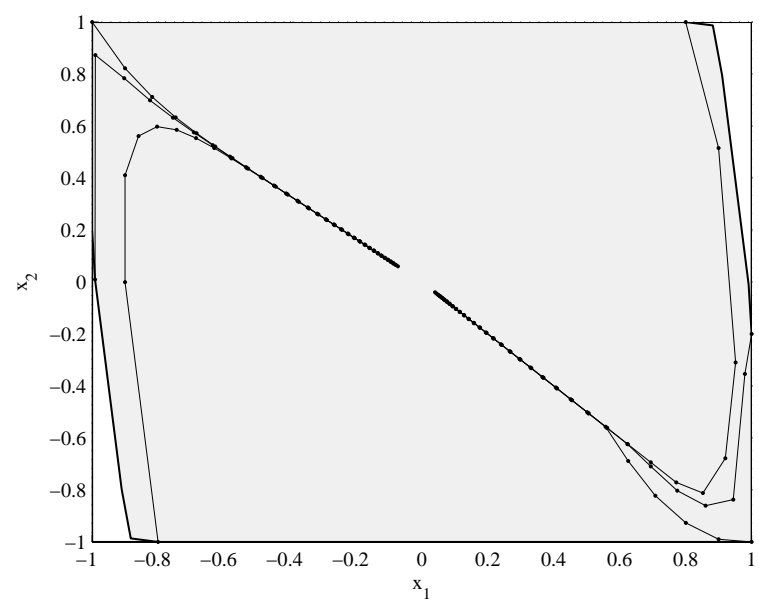

Fig. 2. Maximal invariant set $S_{\max }$, constraint set $S$ and trajectories emanating from some vertices of $S_{\max }$, nonlinear control law case.

The state and input constraint sets $S$ and $U$ respectively are defined by relations

$$
S=\left\{x \in \mathbb{R}^{2}: G_{s} x \leq w_{s}\right\}
$$

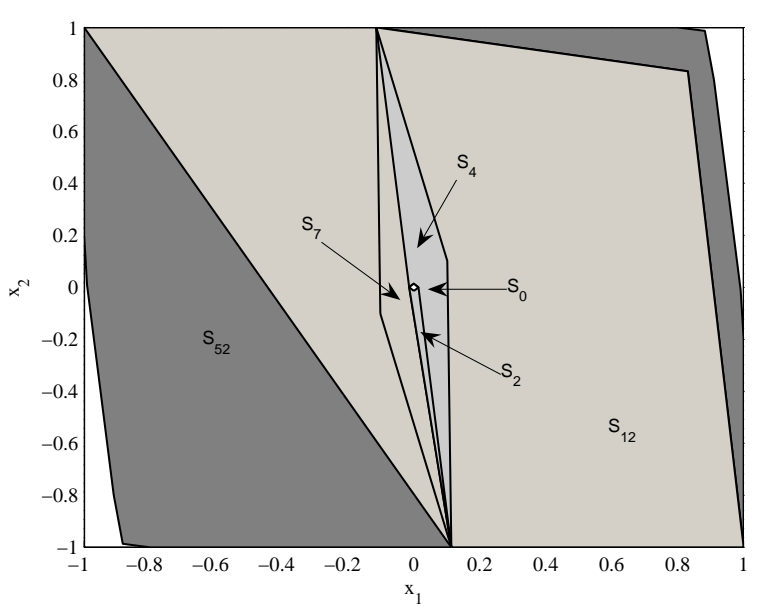

Fig. 3. Sets produced in the expansion procedure, nonlinear control case .

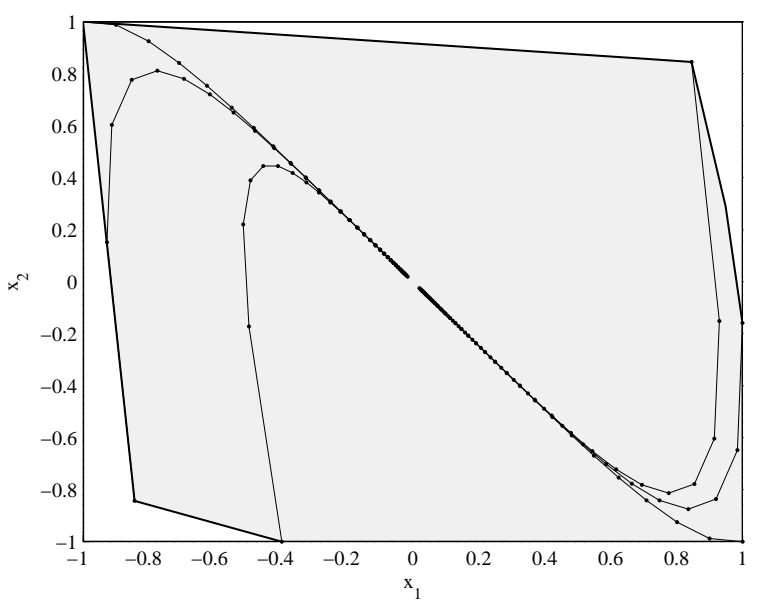

Fig. 4. Maximal invariant set $S_{\max }$, constraint set $S$ and trajectories emanating from some vertices of $S_{\max }$, linear control law case.

where

$$
G_{s}=\left[\begin{array}{cc}
1 & 0 \\
0 & 1 \\
-1 & 0 \\
0 & -1
\end{array}\right], \quad w_{s}=\left[\begin{array}{l}
1 \\
1 \\
1 \\
1
\end{array}\right]
$$

and

$$
U=\left\{u \in \mathbb{R}:-u_{m} \leq u \leq u_{M}\right\}
$$

where $u_{m}=u_{M}=1$. The maximal invariant polyhedral set $S_{\max }$ produced has 14 vertices. The control law is

$$
u(x)=\sum_{i=1}^{14} \lambda_{i}(x) u^{i}
$$

and is computed online at each time instant $t$ by solving the following linear programming problem:

$$
\min _{\lambda_{i}(x(t))}\left\{\left\|A x\left(t ; x_{0}\right)+B \sum_{i=1}^{14} \lambda_{i}\left(x\left(t ; x_{0}\right)\right) u^{i}\right\|_{\infty}\right\}
$$


subject to

$$
\begin{gathered}
x\left(t ; x_{0}\right)=\sum_{i=1}^{14} \lambda_{i}\left(x\left(t ; x_{0}\right)\right) v^{i} \\
\sum_{i=1}^{14} \lambda_{i}\left(x\left(t ; x_{0}\right) \leq \varepsilon\right. \\
\lambda_{i}\left(x\left(t ; x_{0}\right) \geq 0, i=1, . ., 14\right.
\end{gathered}
$$

The initial set $S_{0}$ and the expansion procedure of the controlled invariant set is shown in Fig. 3. It is worth noticing that the maximal invariant set is computed after 52 iterations, i.e 52 points have been added to the convex hull of the initial set, while $S_{\max }$ has only 14 vertices. Finally, by applying the design procedure established above for the linear state feedback control case, a gain matrix

$$
K_{\max }=\left[\begin{array}{ll}
-0.5957 & -0.5875
\end{array}\right]
$$

is computed. The set $S_{\max }$ has 8 vertices. This set together with the trajectories starting from some of its vertices is shown in Fig. 4. It is clear that this method gives better results than the one used in [24].

\section{CONCLUSIONS}

In this paper, a method for the determination of the maximal controlled invariant set which is also a domain of attraction under a suitable state-feedback control law, has been established. The method applies to both continuous-time and discrete-time linear systems with polyhedral constraints. The convergence of the algorithm is guaranteed. In order to illustrate the performance of the method, an example, studied also in [24], has been chosen. It has been shown that the proposed method provides better approximation of the maximal invariant set for the case of nonlinear control laws and a larger invariant set and a simpler controller for the case of linear state-feedback.

\section{REFERENCES}

[1] D.Q. Mayne, J.B. Rawlings, C.V. Rao and P.O.M. Scokaert, "Constrained Model Predictive Control: Stability and Optimality", Automatica, Vol. 36, pp. 789-814, 2000.

[2] F. Blanchini, "Set Invariance in Control", Automatica, Vol. 35, pp. 1747-1767, 1999.

[3] H. Michalska, D.Q. Mayne, "Robust receding horizon control of constrained nonlinear systems", "IEEE Transactions on Automatic Control, Vol. 38, pp. 1623-1633, 1993

[4] B.D. O'Dell and E.A. Misawa, "Semi-Ellipsoidal Controlled Invariant Sets for Constrained Linear Systems", Journal of Dynamic Systems, Measurements and Control, Vol. 124, pp. 98-103, 2002.

[5] P. Gutman and M. Chwikel "An Algorithm to Find Maximal State Constraint Sets for Discrete-Time Linear Dynamical Systems with Bounded Controls and States" IEEE Transactions on Automatic Control, vol. 32, pp. 251-254, 1987.

[6] E.G. Gilbert and K.T. Tan, "Linear Systems with State and Control Constraints: The Theory and Application of Maximal Output Admissible Sets", IEEE Transactions on Automatic Control, vol. 36, pp. 1008-1020, 1991 .

[7] C.E.T. Dorea and J.C. Hennet, "Computation of Maximal Admissible Sets of Constrained Linear Systems" in 4th IEEE Mediterranean Symposium on New Directions in Control and Automation, 1996.

[8] C.E.T. Dorea and J.C. Hennet, ”(A,B)-Invariant Polyhedral Sets of Linear Discrete-Time Systems", Journal of Optimization Theory and Applications, Vol. 103, pp. 521-542, 1999.
[9] J.B. Lasserre, "Reachable, Controllable Sets and Stabilizing Control of Constrained Linear Systems", Automatica, Vol. 29, pp. 531-536, 1993.

[10] D. Limon, T. Alamo and E.F. Camacho, "Enlarging the domain of attraction of MPC controllers", Automatica, Vol. 41, pp. 629-635, 2005.

[11] F. Blanchini, F. Mesquine and S. Miani, "Constrained Stabilization with an Assigned Initial Condition Set", International Journal of Control, vol. 62, pp. 601-617, 1995

[12] T. Hu and Z. Lin, "On Enlarging the Basin of Attraction for Linear Systems under Saturated Linear Feedback", Proceedings of the Amercan Control Conference, 2000.

[13] B. Kouvaritakis, M. Cannon, A. Karas, B. Rohal-Ilkiv and B. Belavy, "Asymmetric constraints with polytopic sets in MPC with aplication to coupled tanks system", International journal of Robust and Nonlinear Control, Vol. 14, pp. 341-353, 2004.

[14] D. Henrion, S. Tarbouriech and V. Kucera, "Control of linear systems subject to input constraints: a polynomial approach", Automatica, Vol. 37 , pp. 597-604, 2001.

[15] T.B. Blanco and B. De Moor, "Polytopic invariant sets for continuoustime systems", In Proceedings of the European Control Conference, 2007.

[16] G. Bitsoris, "On the positive invariance of polyhedral sets for discretetime systems", Systems and Control Letters, Vol. 11, pp. 243-248, 1988.

[17] E. Gravalou, "Control of constrained dynamical systems", Phd Thesis, University of Patras, 1995.

[18] L. Farina and L. Benvenuti, "Invariant polytopes of linear systems", IMA Journal of Mathematical Control and Information, Vol. 15, pp. 233-240, 1998.

[19] G.Bitsoris, "Existence of positively invariant polyhedral sets for continuous-time linear systems", Control-Theory and Advanced Technology, Vol. 7, pp. 407-427, 1991.

[20] E.B. Castelan and J.C. Hennet, "On Invariant Polyhedra of Continuous-Time Linear Systems", IEEE Transactions on Automatic Control, Vol. 38, pp. 1680-1685, 1993.

[21] F. Blanchini and S. Miani, "Set-theoretic methods in control", Birkhauser, 2007.

[22] P.O. Gutman and M. Cwikel, "Admissible Sets and Feedback Control for Discrete-Time Linear Dynamical Systems with Bounded Controls and States", IEEE Transaction on Automatic Control, Vol. 31, pp.373376, 1986.

[23] G. Bitsoris and E. Gravalou, "Design Techniques for the Control of Discrete-Time Systems Subject to State and Control Constraints", IEEE Transactions on Automatic Control, Vol. 44, pp. 1057-1061, 1999.

[24] F. Blanchini, S. Miani and C. Savorgnan, "Dynamic augmentation and complexity reduction of set-based constrained control", In Proceedings of the 17th IFAC World Congress, 2008. 